\title{
REVIEWERS FOR VOLUME 16
}

In addition to the editorial board, I have been fortunate to have the support of many individuals who have contributed to the review process this past year. I would like to thank them for their time and thoughtful reviews.

Karen Abram

Don Bersoff

Eugene Borgida

Angela Browne

Dewey Cornell

Frank Dane

David Faigman

Diane Follingstad

Sol Fulero

Gail Goodman

Craig Haney

Steve Hart

Geoff Haugaard

Larry Heuer

Norb Kerr

Margaret Kovera

Larry Kramer

Dennis Krebs

Elizabeth Luus

James Luginbuhl

Roy Malpass

\author{
Reid Meloy \\ Robert Meyer \\ Cathy McFarland \\ Michael Perlin \\ Don Read \\ Julian Roberts \\ Dick Rogers \\ David Ross \\ Karen Saywitz \\ David Scherer \\ Vincent Sacco \\ Kathleen Sheridan \\ Vicki Smith \\ Loretta Stalans \\ Nicholas Spanos \\ Neil Vidmar \\ David Wexler \\ Roselle Wissler \\ Dan Yarmey \\ John Yuille
}

